\title{
Flow Simulation of Supersonic Inlet with Bypass Annular Duct
}

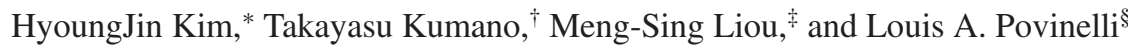 \\ NASA John H. Glenn Research Center at Lewis Field, Cleveland, Ohio 44135 \\ and \\ Timothy R. ConnersI \\ Gulfstream Aerospace Corporation, Savannah, Georgia 31402 \\ DOI: $10.2514 / 1.49028$
}

\begin{abstract}
A relaxed isentropic compression supersonic inlet is a new concept that produces smaller cowl drag than a conventional inlet, but incurs lower total pressure recovery and increased flow distortion in the (radially) outer flowpath. A supersonic inlet comprising a bypass annulus to the relaxed isentropic compression inlet dumps out airflow of low quality through the bypass duct. A reliable computational fluid dynamics solution can provide considerable useful information to ascertain quantitatively relative merits of the concept, and further provide a basis for optimizing the design. For a fast and reliable performance evaluation of the inlet performance, an equivalent axisymmetric model whose area changes accounts for geometric and physical (blockage) effects resulting from the original complex three-dimensional configuration is proposed. In addition, full three-dimensional calculations are conducted for studying flow phenomena and verifying the validity of the equivalent model. The inlet-engine coupling is carried out by embedding numerical propulsion system simulation engine data into the flow solver for interactive boundary conditions at the engine fan face and exhaust plane. It was found that the blockage resulting from complex three-dimensional geometries in the bypass duct causes significant degradation of inlet performance by pushing the terminal normal shock upstream.
\end{abstract}

\section{Nomenclature}

$\begin{array}{ll}A & =\text { area } \\ \mathrm{AIP} & =\text { aerodynamic interface plane, engine fan face } \\ B & =\text { blockage } \\ \mathrm{TPR} & =\text { total pressure recovery } \\ \mathrm{MFR} & =\text { mass-flow rate }, \dot{m} \\ P_{b} & =\text { backpressure }\end{array}$

\section{Introduction}

$\mathbf{T}$ HROUGH a supersonic inlet, a supersonic freestream flow is decelerated and diffused to subsonic speed before entering the engine. A shock train, a series of shock waves during compression, can occur in the supersonic region of the inlet and is eventually terminated by a normal shock. The location of the shock train categorizes the type of inlets as internal, external, or mixed compressions.

Mass-flow rate and total pressure recovery are two basic performance parameters of a supersonic inlet. Another important factor for supersonic inlet designs is the cowl drag, which is primarily the wave drag due to the difference between the cowl angle and freestream flow angle. In conventional external and mixed compression inlet design practice, all shock waves from the external forebody surface

Presented as Paper 2010-480 at the 48th AIAA Aerospace Science Meeting, Orlando, FL, 4-7 January 2010; received 22 January 2010; revision received 17 August 2010; accepted for publication 17 August 2010. This material is declared a work of the U.S. Government and is not subject to copyright protection in the United States. Copies of this paper may be made for personal or internal use, on condition that the copier pay the $\$ 10.00$ percopy fee to the Copyright Clearance Center, Inc., 222 Rosewood Drive, Danvers, MA 01923; include the code 0748-4658/11 and $\$ 10.00$ in correspondence with the CCC.

*NPP (NASA Postdoctoral Program) Senior Fellow; currently Senior Researcher, Ohio Aerospace Institute, 22800 Cedar Point Road, Cleveland, $\mathrm{OH}$ 44142. Senior Member AIAA.

${ }^{\dagger}$ NPP Fellow. Member AIAA.

${ }^{\ddagger}$ Senior Technologist. Associate Fellow AIAA.

${ }^{\S}$ Senior Technologist. Fellow AIAA.

TPrincipal Engineer, Propulsion, Preliminary Design Department, Mail Stop R-07. Associate Fellow AIAA are focused to the cowl lip, thereby resulting in low flow spillage, good total pressure recovery, and flow quality in subsonic diffuser. The conventional design determines cowl angle according to the local flow angle. A larger local flow angle near the cowl by higher compression causes a larger cowl drag. Recently, Conners and Howe [1] proposed the relaxed isentropic compression inlet, a novel concept for supersonic inlet with remarkably reduced cowl drag. It is a combination of straight and curved surfaces: a straight cone in the forward part of the inlet followed by a smooth curved surface providing an isentropic compression. Unlike conventional designs, only the first oblique shock wave from the cusp of the cone is focused on the cowl lip, and the isentropic compression waves from the curved surface intersect and modulate the terminal normal shock. The flow at the outer radius region near the cowl experiences less compression than conventional inlets and hence has less turning caused by compression. This allows a reduced cowl angle with respect to the freestream flow, hence enabling a significant reduction of cowl drag.

A demerit of the relaxed isentropic compression inlet is that the flow distortion increases, especially in the outer flowpath near the cowl because of the strong velocity gradient resulting from the uneven compression between inner and outer flowpaths. The stronger normal shock in the outer flowpath also causes reduction in total pressure recovery. In addition, the reduced cowl angle requires a rapid turning angle in the centerbody shoulder region in order to give an adequate subsonic diffuser area distribution. This rapid turning induces a thicker boundary layer in the subsonic diffuser. Flow control devices, such as micro vortex generators, may be necessary to improve the boundary-layer health in the subsonic diffuser [2].

The annulus bypass flow concept was originally proposed for attenuating the sonic boom strength. The bypass concept, however, has an unintended benefit to remedy the aforementioned drawbacks of the relaxed compression inlet by disposing the flow of undesirable features.

In this study we conduct flow simulation of a three-dimensional configuration that includes a relaxed isentropic compression inlet, a bypass flow annulus, and a plug nozzle. The full three-dimensional configuration also includes struts, gearbox fairing, and flow guide vanes in the bypass annular duct for structural and aerodynamic purposes. We also include the effects of a core engine to complete the 
simulation of the entire propulsion system. Inclusion of the core engine, the Rolls-Royce Tay 651 engine** modeled by the Numerical Propulsion System Simulation (NPSS), provides proper and realistic boundary conditions for imposing thermodynamic variables at the engine fan and exhaust faces. Flow simulations are made for the axisymmetric and the full three-dimensional configurations; the former is an equivalent geometry derived by accounting for the geometric and physical blockage effects based on the full threedimensional configuration.

In the following section, more details of the bypass inlet configuration are presented. Section III describes elements for flow analysis, of special interest is concerned with methodologies for imposing boundary conditions for inlet-engine coupling and for efficiently generating approximate cane curves. Numerical results on the axisymmetric and full three-dimensional configurations are described in Sec. IV. Finally, concluding remarks are given in Sec. $\underline{\text {. }}$.

\section{Supersonic Bypass Inlet Configuration}

\section{A. Overall Configuration}

The full three-dimensional engine nacelle configuration includes the inlet forebody, a bypass flow annulus and a plug nozzle, as illustrated in Fig. 1a. The core engine is the Rolls-Royce Tay 651 engine, depicted in Fig. 2, which is a turbofan engine with a bypass ratio of 3.1 and a maximum takeoff thrust of $15,400 \mathrm{lbf}$. Note that the bypass ratio is for the core engine and should not be confused with the bypass annular duct of the inlet.

\section{B. Struts and Gearbox Fairing}

Figure $1 \mathrm{~b}$ shows the shape of the gearbox fairing and struts in the bypass annular duct. There are nine thin-plate struts in the front and nine relatively thick struts in the rear. Struts are installed there to guide the bypass flow and also for structural purposes. The shapes of struts were designed in a one-dimensional way to locate the throat of the annular duct near the leading edge of the rear struts and to accelerate flow into supersonic speed downstream of the throat. An outer view of the CAD model for the whole geometry is shown in Fig. 1c.

\section{Axisymmetric Configuration}

As a preliminary step before simulating the full three-dimensional geometry, an axisymmetric configuration is generated and analyzed. The inner cowl wall of the full model is modified so that the section area distribution of the axisymmetric bypass duct is the same as the three-dimensional bypass duct shown in Fig. 3 and will be referred to as AS-1 (axisymmetric configuration 1). The rear nozzle part of the duct does not follow the area distribution in order not to alter shape of the outer cowl wall, which would not change the inlet flow patterns because the bypass duct is choked for the flow conditions considered here. The axisymmetric configuration includes the inlet, bypass annular duct, engine face and exit, and plug nozzle.

\section{Methodology}

\section{A. Flow Solver}

TAS-Flow [3], a finite volume unstructured-grid Navier-Stokes solver, is used for flow simulations. The Reynolds-averaged NavierStokes (RANS) equations are discretized by the cell-vertex finite volume method. Control volumes are nonoverlapping dual cells constructed around each node. Each edge connecting two nodes is associated with an area vector of the control surface, at which flow fluxes are computed. To enhance the accuracy of the scheme, a linear reconstruction of the primitive gas dynamic variables inside the control volume is used in conjunction with a limiter [4]. The inviscid flux is computed using the Harten-Lax-van Leer-Einfeldt-Wada (HLLEW) approximate Riemann solver [5]. Turbulence effects are considered by the Spalart-Allmaras one-equation turbulence model

**Data available online at http://www.rolls-royce.com/deutschland/en/ products/tayspeydart.htm [retrieved 16 September 2010].

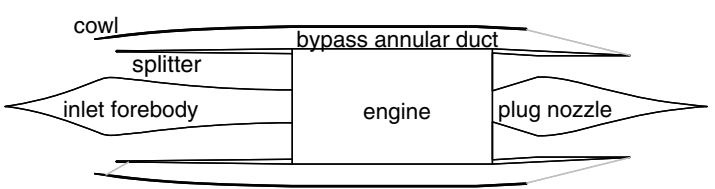

a) Supersonic bypass inlet configuration

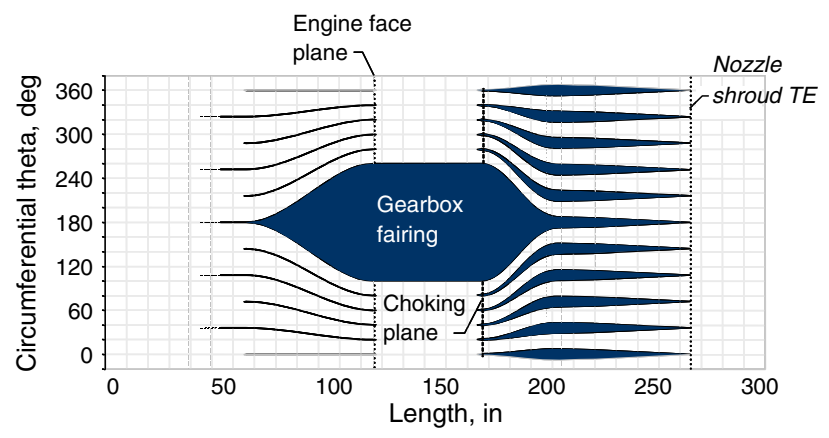

b) Shapes of struts and gearbox fairing in the bypass annular duct

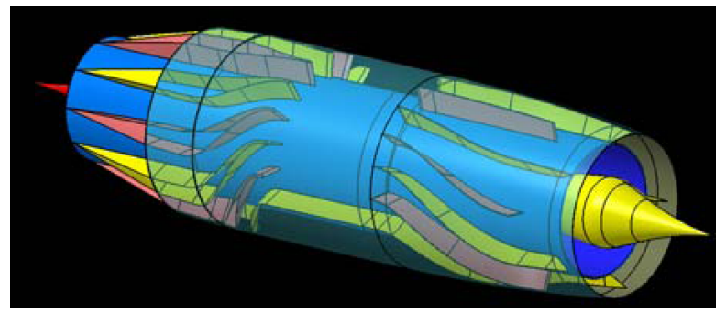

c) CAD model of the full configuration

Fig. 1 Full configuration of the supersonic bypass nacelle, showing struts and gearbox fairing (in the bottom portion).

[6]. Since no massive flow separation appears for the flow conditions in the present study, RANS simulation with the Spalart-Allmaras one-equation turbulence model is deemed to be adequate for the flow simulations. For the time integration, the lower/upper symmetric Gauss-Seidel (LU-SGS) implicit method is adopted [7]. Parallel processing is made by domain decomposition and MPI communication library. Implementation details and validation of the flow solver can be found in [3] .

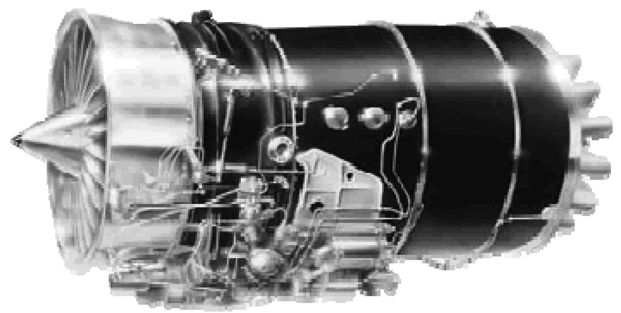

Fig. 2 Rolls-Royce Tay 651 engine.

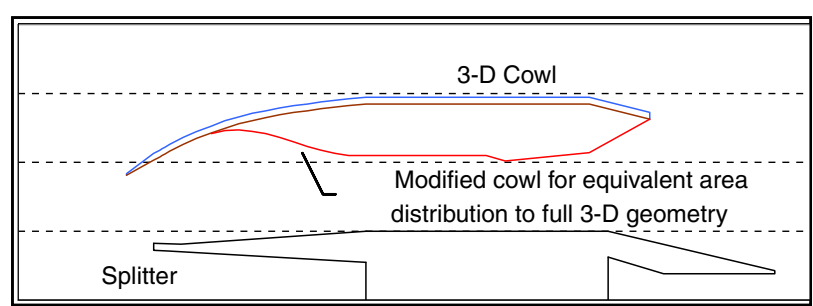

Fig. 3 AS-1: the modified shape of the cowl inner wall having section area equivalent to the full three-dimensional geometry for axisymmetric flow analysis. 


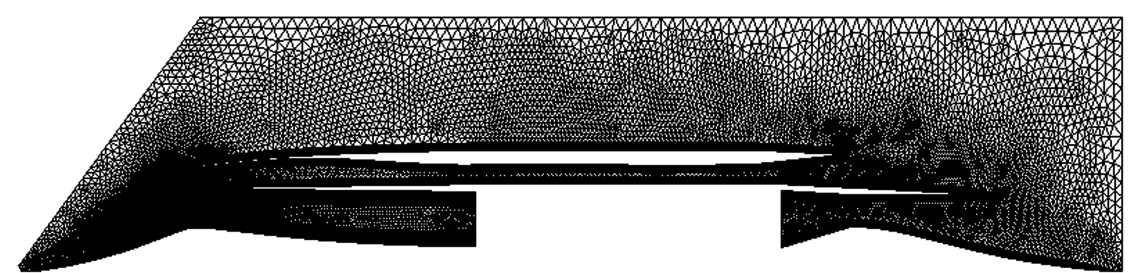

Fig. 4 Computational grid for axisymmetric configuration AS-1 (numbers of nodes: 152,040).

\section{B. Grid Generation}

For the axisymmetric configuration, we built a two-dimensional hybrid grid: quadrangular cells near the viscous walls and triangles for the remaining computational domain. The two-dimensional grid is rotated for a small angle about the $x$ axis to build a pie-shaped three-dimensional grid. Figure $\underline{4}$ shows the computational grid for the axisymmetric configuration.

For the three-dimensional full geometry with struts in the bypass duct, a surface mesh shown in Fig. 5 is generated directly on stereolithography (STL) data [8]. A hybrid mesh is generated with prism layers near viscous walls, tetrahedral cells in the remaining computational domain, and pyramid cells in between when necessary. Generation of the hybrid mesh is made by the advancing front/layer method using MEGG3D [9].

\section{Boundary Conditions}

Flow conditions are for a supersonic cruise flight at freestream Mach 1.7 and altitude of 45,000 ft. As for the engine fan face boundary conditions, we extrapolate the density and velocity components from inside of computational domain and impose the backpressure to adjust the mass-flow rate. At the engine exhaust, total pressure and total temperature are imposed as inflow conditions for the aft section of the engine core in the computation domain.

For evaluation of the total axial force combining drag and thrust forces for the three-dimensional configuration, boundary conditions at the fan face and engine exhaust are imposed based on engine cycle analysis results. To this end, an engine simulation code, Numerical Propulsion System Simulation (NPSS) [10] is employed to model the Tay 651 engine. NPSS is a multidisciplinary analysis environment for aerospace propulsion systems and allows an efficient analysis of aircraft engine performances. In this study, NPSS provides steadystate performance and thermodynamic characteristics of the Tay 651 engine.
For the inlet-engine coupling, the following approach is adopted in the present study:

1) For fixed values of the inlet total pressure recovery (TPR) in a proper range of [0.90, 0.97], for instance, run NPSS to find the engine mass-flow rate for each TPR and its corresponding engine exhaust conditions.

2) Make polynomial fittings for the mass-flow rate, nozzle pressure ratio and exhaust total temperature as functions of inlet TPR.

3) Embed the polynomials into the flow solver so that boundary conditions are imposed depending on the inlet TPR on the fly if inletengine coupling is needed.

4) Run the flow solver with the boundary conditions just determined. Adjust the backpressure and nozzle throat area on the fly to match the target mass-flow rate.

Figure 6 shows the engine data generated from NPSS for imposing boundary conditions as a function of inlet TPR in the flow simulations. The engine mass-flow rate is proportional to the inlet TPR and can be exactly fitted by a linear function. The nozzle pressure ratio and exhaust total temperature are also proportional to the TPR and both show an almost linear variation.

When a target mass-flow rate through the engine face is given from the linear relation of TPR vs mass-flow rate (MFR) in Fig. 6a, the actual mass-flow rate can be matched to a target value by periodically updating the static pressure $p$ at the engine face boundary using the following iterative relation [11]:

$$
p^{n+1}=p^{n}+0.2\left(\frac{\dot{m}_{\mathrm{AIP}}^{n}}{\dot{m}_{\mathrm{target}}}-1\right)
$$

where $n$ represents the iteration level.

At the engine exhaust, the throat area of the plug nozzle is adjusted in order to match the engine exhaust mass-flow rate. First, the required throat area is calculated from the engine exhaust total conditions and target mass-flow rate [12]:

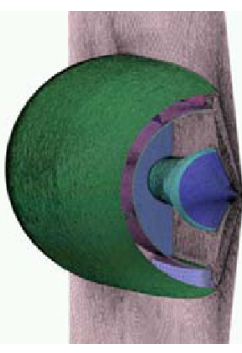

a) Front part

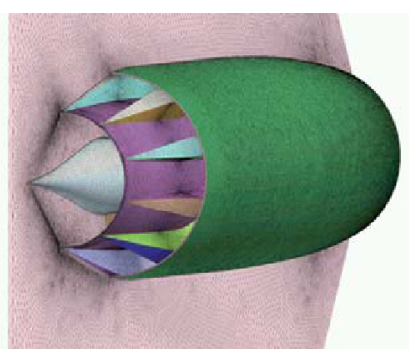

b) Rear part

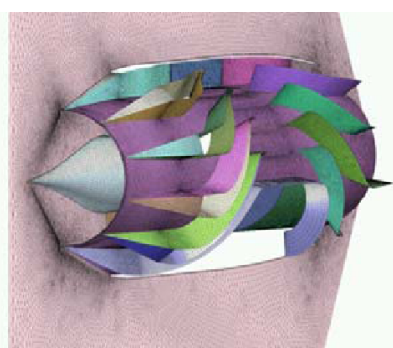

c) Struts and fairing

Fig. 5 Computational grid for the full three-dimensional configuration (number of nodes: 7,214,163). 


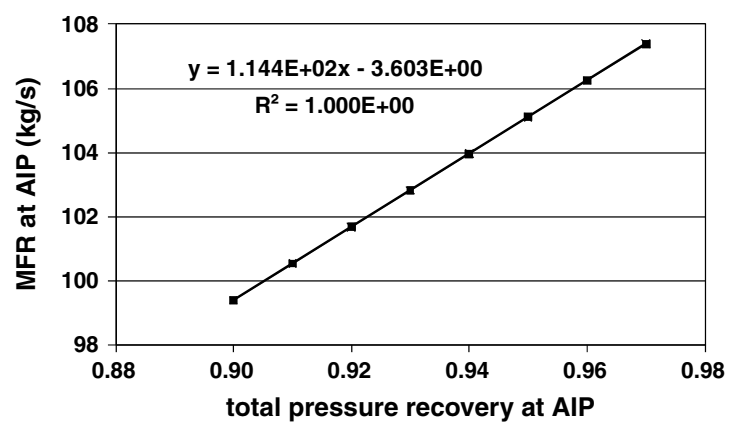

a) TPR vs. MFR

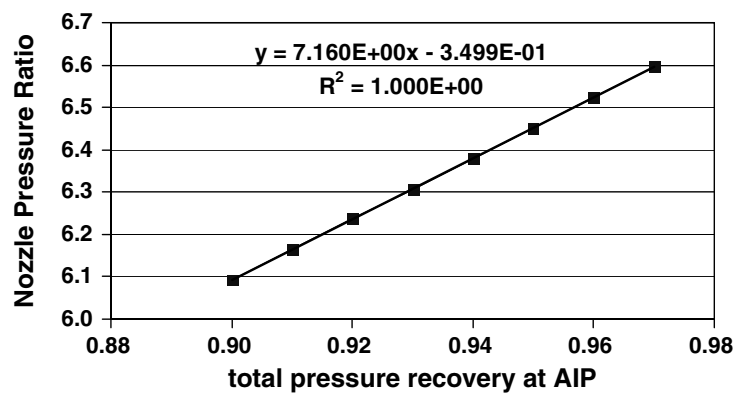

b) TPR vs. nozzle pressure ratio

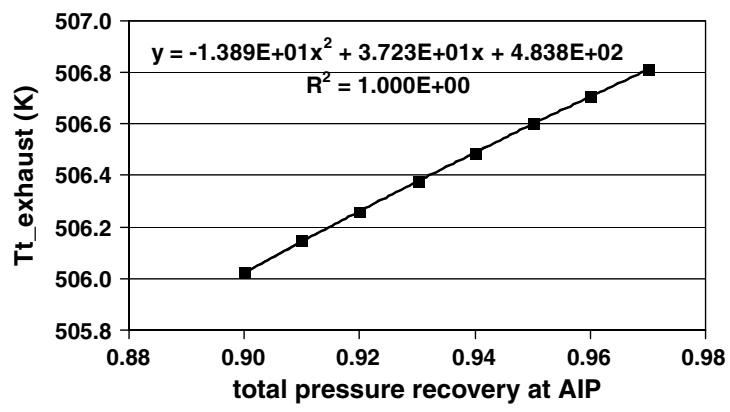

c) TPR vs. engine exhaust total temperature

Fig. 6 Engine data for the variation of inlet total pressure recovery at the flight condition.

$$
A^{*}=\frac{1}{K} \frac{\sqrt{T_{t}}}{p_{t}} \dot{m}
$$

where

$$
K=\sqrt{\frac{\gamma}{R}\left(\frac{2}{\gamma+1}\right)^{\frac{\gamma+1}{\gamma-1}}}
$$

which is a function of gas properties, the gas constant $R$ and ratio of specific heat $\gamma$. In this study, it was assumed that engine exhaust gas has the same gas property as the freestream air: $R=287 \mathrm{~J} / \mathrm{kg} \mathrm{K}$ and $\gamma=1.4$.

The throat area calculated with Eq. (2) would result in a slightly lower mass-flow rate than required because of effects of the boundary-layer thickness. The actual throat area needs to be determined iteratively on the fly through a relaxation similar to Eq. (1):

$$
A^{n+1}=A^{n}+0.2\left(\frac{\dot{m}_{\text {target }}}{\dot{m}_{\text {exhaust }}^{n}}-1\right)
$$

The surface grid is modified according to the updated throat area. The volume grid points are then adjusted accordingly using a spring analogy approach. A spring coefficient for each edge is defined as being inversely proportional to edge lengths to prevent tangled elements. Computational cost for the spring analogy is negligible compared to that of the flow analysis.
D. Efficient Generation of Cane Curves for the Full Three-Dimensional Configuration

The cane curves are manifestation of inlet performance in terms of mass-flow rate versus total pressure recovery at the aerodynamic interface plane (AIP). Generation of cane curves is essential for evaluation of inlet performance. For conventional single-passage supersonic inlets without bypass duct, inlet performance is a function of inlet geometry, freestream flow conditions, and mass-flow rate (or backpressure) at the AIP. Therefore, a cane curve built with flow analysis or wind tunnel testing data can be directly adopted for inletengine matching. However, for a bypass inlet, the performance is also affected by the blockage of the bypass duct both from the change of section area of complex flowpath and viscous flow physics throughout.

Generating the cane curve for the full three-dimensional inlet configuration by analyzing the full geometry at various backpressure conditions is a very time-consuming and expensive task. It would be very helpful if axisymmetric flow simulation can be used for building cane curves as if it were an axisymmetric single-passage inlet, provided that this approach (model) includes all essential physics (primarily the blockage effect in this case). In the previous subsection for mesh generation, AS-1, the axisymmetric configuration with equivalent section area to the full three-dimensional geometry is introduced. However, the AS-1 does not include boundary-layer thickness or other additional blockage due to the flow physics in the highly curved flowpath. One can expect that the AS-1 and full

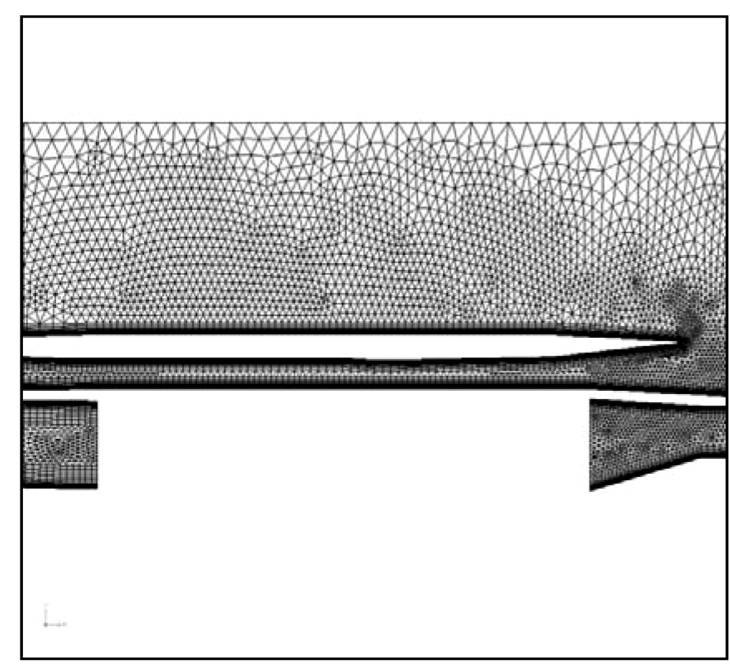

a) Initial configuration (AS-1)

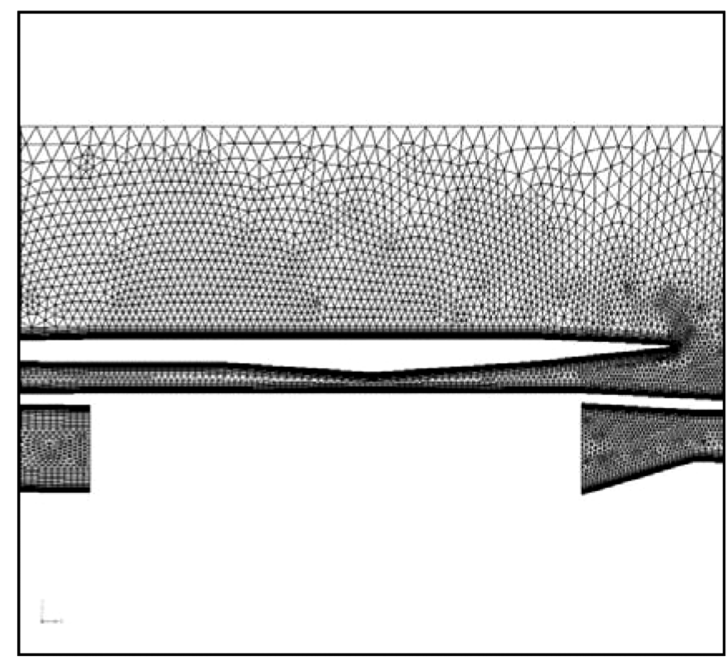

b) A modified configuration for blockage matching (AS-2)

Fig. 7 Modification of the minimum section area of the axisymmetric configuration for blockage matching in the bypass duct. 

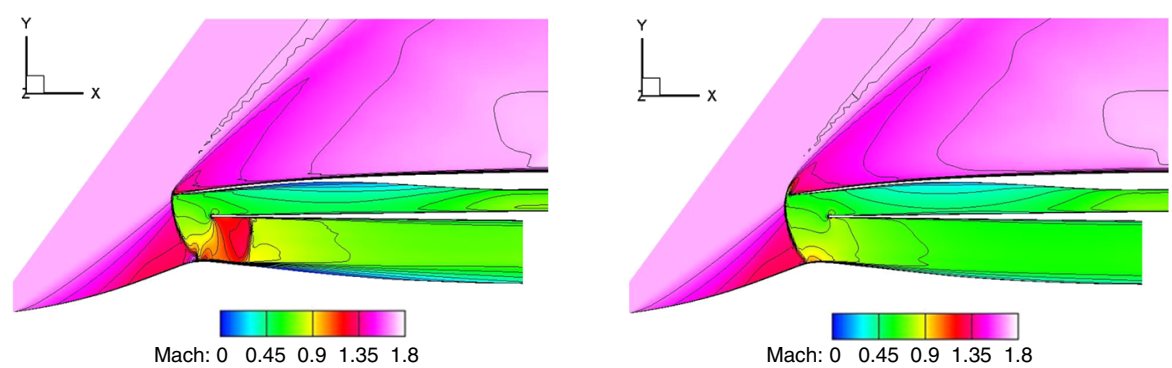

a) $P_{b} / P_{\infty}=3.40$

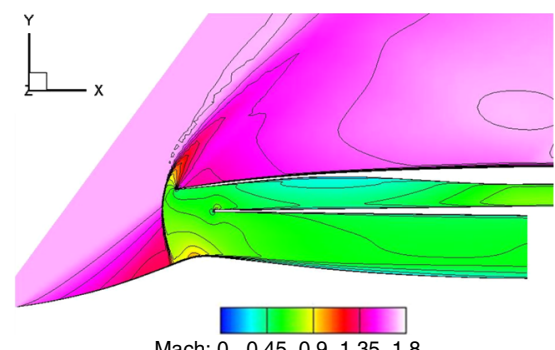

b) $P_{b} / P_{\infty}=3.70$

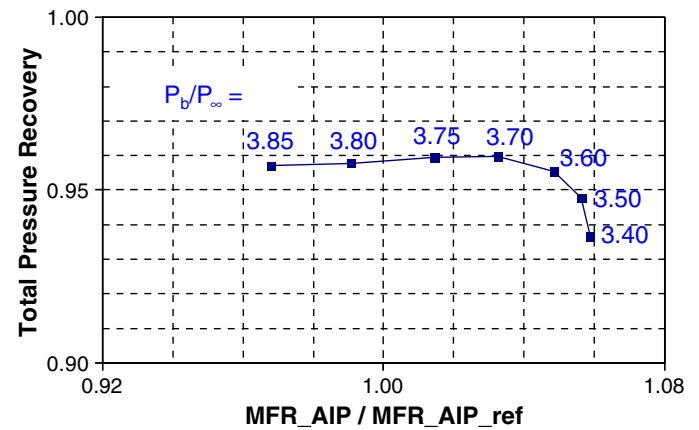

c) $P_{b} / P_{\infty}=3.85$

d) Cane curve for AS-1

Fig. 8 Representative Mach contours and the cane curve for the axisymmetric configuration AS-1.

three-dimensional configurations would show performance differences, although they have the same inlet geometry and section area distribution in the bypass duct. Consideration of the section area alone in the axisymmetric analysis is not sufficient for evaluating performance of the full three-dimensional bypass inlet configuration; the additional blockage should also be taken into account.

Here, we introduce an efficient way for approximating cane curves for bypass inlets with complex flowpath. For the same freestream conditions and inlet geometry such as forebody, cowl, and splitter shown in Fig. 1a, inlet performances are functions of backpressure at the AIP $\left(p_{b}\right)$ and blockage through the bypass $(B)$ :

$$
\mathrm{TPR}_{\mathrm{AIP}}=f_{1}\left(p_{b}, B\right), \quad \mathrm{MFR}_{\mathrm{AIP}}=f_{2}\left(p_{b}, B\right)
$$

We assume that $B$ can be modeled in an axisymmetric fashion by changing the minimum area of the bypass duct. Then, the inlet performance can be uniquely defined by specifying the backpressure at the AIP and the minimum section area through the bypass duct. The procedure is as follows:

1) For a certain backpressure, conduct flow simulation for the full three-dimensional geometry to get $\mathrm{TPR}_{\mathrm{AIP}_{3-\mathrm{D}}}$ and $\mathrm{MFR}_{\mathrm{AIP}_{3-\mathrm{D}}}$.

2) At the same backpressure, solve the equivalent axisymmetric configuration by adjusting the minimum area of the bypass duct, and select the minimum area that (closely) matches $\mathrm{TPR}_{\mathrm{AIP}_{3-\mathrm{D}}}$ and $\mathrm{MFR}_{\mathrm{AIP}_{3-\mathrm{D}}}$.

3) If a linear variation of the blockage is desired with a two-point fit, repeat steps 1 and 2 once more for another backpressure condition.

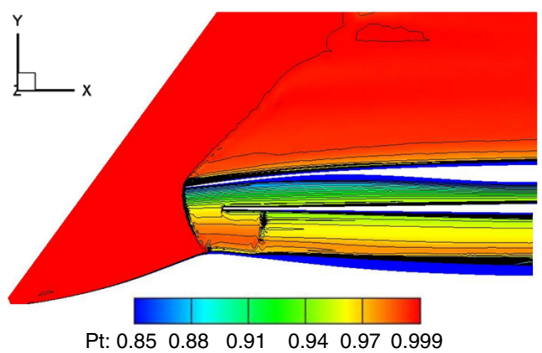

a) $P_{b} / P_{\infty}=3.40$

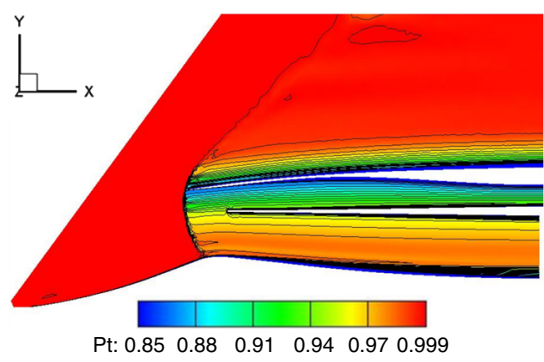

b) $P_{b} / P_{\infty}=3.70$

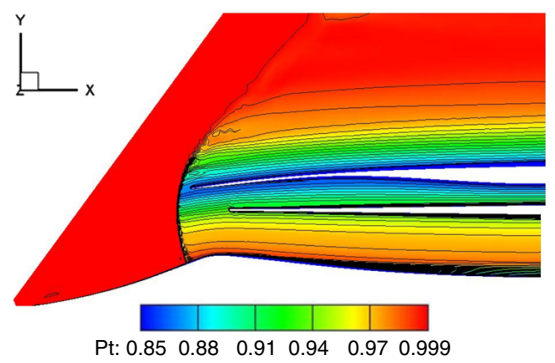

c) $P_{b} / P_{\infty}=3.85$

Fig. 9 Normalized total pressure contours for the axisymmetric configuration AS-1. 


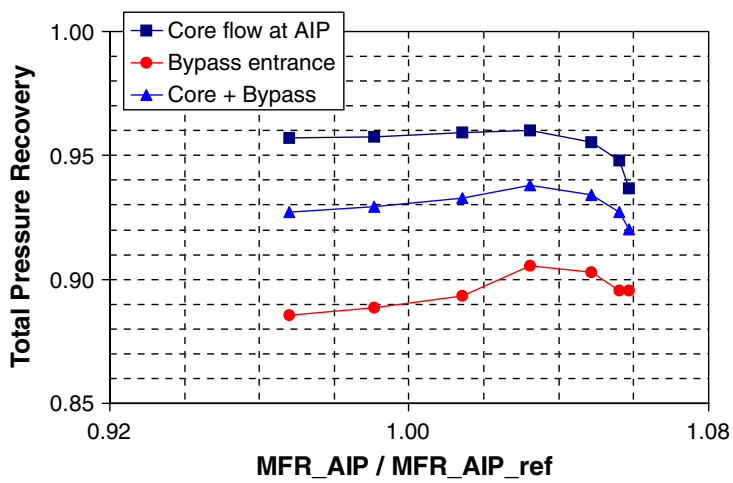

a) Total pressure recovery at AIP and bypass entrance

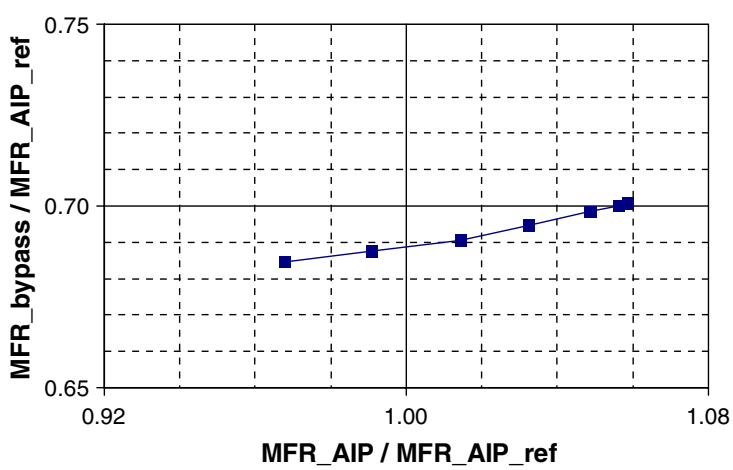

b) Relation between mass flow rates at AIP and bypass duct

Fig. 10 Results from simulation for the axisymmetric configuration AS-1.

4) Perform flow simulations for the axisymmetric configuration with the constant or linear minimum area change and vary backpressures to build an approximate cane curve.

5) Validate at some selected points on the cane curve by solving the full three-dimensional geometry with the corresponding backpressures.

Here, we are making another assumption that the blockage $B$ in the bypass duct is constant or varies linearly as the mass-flow rate at the AIP changes. The above approach can be used to show the overall trend of bypass inlet performances and would be especially helpful in

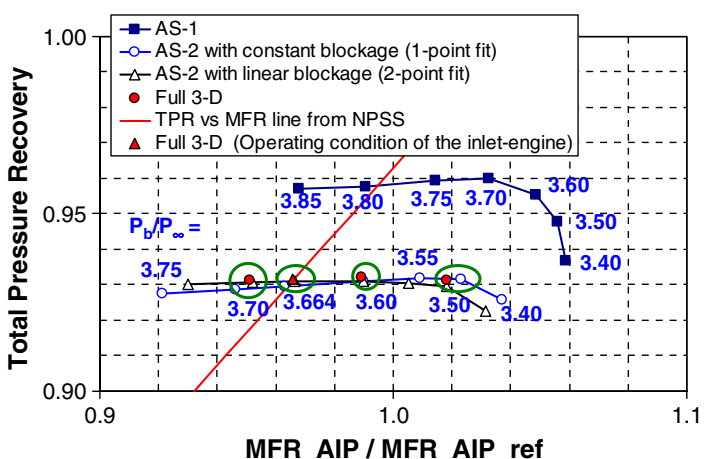

Fig. 11 Comparison of cane curves for axisymmetric and full threedimensional configurations. Figures represent normalized backpressure values. For AS-2 with the one-point fit, the blockage is matched at a normalized backpressure of 3.60. For the two-point fit, the additional point is backpressure of 3.70 .

the preliminary design phase of engine-bypass inlet coupling. The axisymmetric configuration with the matched blockage in the bypass duct will be called AS-2 hereafter. Figure 7 compares grids of original (AS-1) and modified (AS-2) axisymmetric configurations for the bypass blockage matching. It should be noted that the actual duct shape of AS-2 changes for different backpressure conditions if a linear blockage matching is applied.

\section{Results}

A. Axisymmetric Configuration 1

The full three-dimensional configuration has struts and fairing in the bypass duct, which will cause blockage effects by boundary-layer thickness and complex three-dimensional phenomena due to turning and obstruction of flows, possibly producing shock waves. Before looking into the complex full three-dimensional configuration, we first examined AS-1, the axisymmetric configuration with the same sectional area distribution in the bypass duct as the full threedimensional configuration.

In Fig. 8, representative Mach number contours and a performance curve are depicted for AS-1 at the supersonic cruise condition of $M_{\infty}=1.7$ and altitude of $45,000 \mathrm{ft}$. The Mach number is about 1.3 at the foot of the normal shock wave and increases as it goes outward to the cowl lip, which is one of the main characteristics of the relaxed compression inlet.
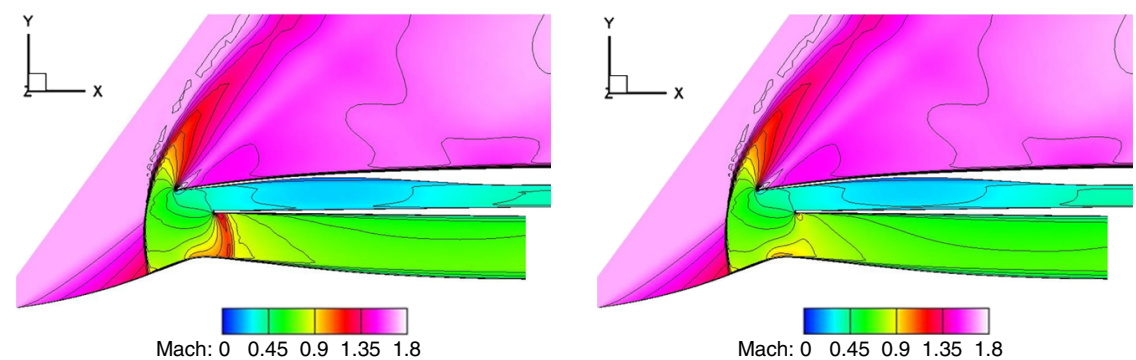

a) $P_{b} / P_{\infty}=3.40$

b) $P_{b} / P_{\infty}=3.50$
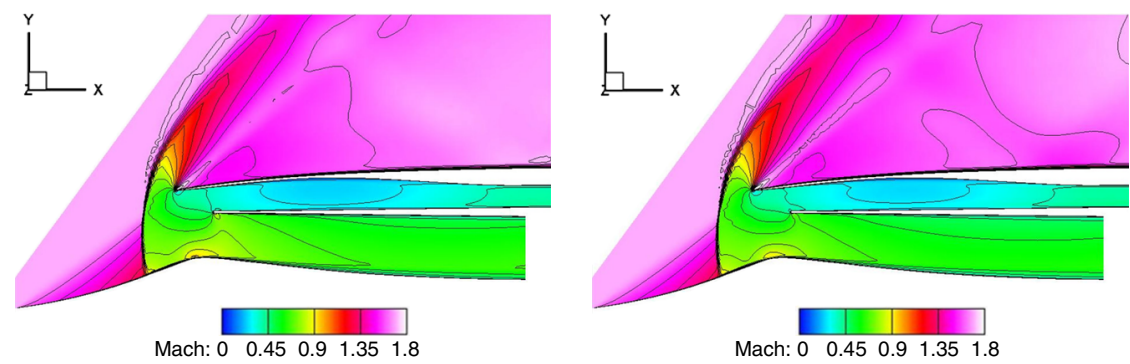

c) $P_{b} / P_{\infty}=3.60$

d) $P_{b} / P_{\infty}=3.70$

Fig. 12 Mach contours for AS-2 with a two-point fit: the axisymmetric configuration with the linear blockage (refer to Fig. 11). 


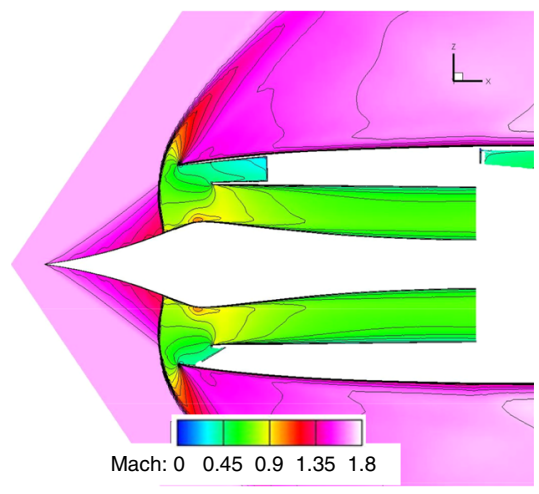

a) $P_{b} / P_{\infty}=3.50$

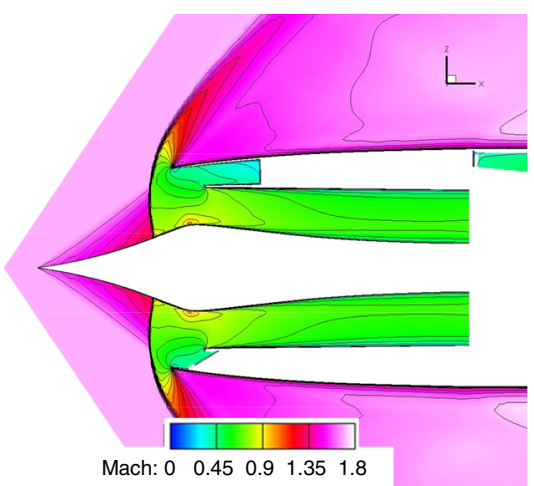

b) $\boldsymbol{P}_{b} / \boldsymbol{P}_{\infty}=\mathbf{3 . 6 0}$

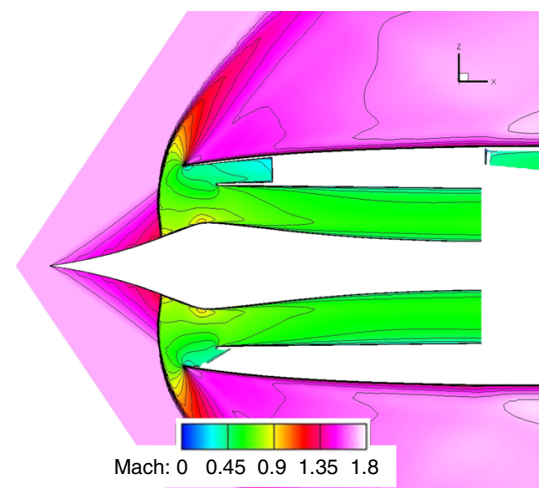

c) $P_{b} / P_{\infty}=3.70$

Fig. 13 Mach contours for the full three-dimensional configuration (refer to Fig. 11).

The reference mass-flow rate at the AIP is set as $235 \mathrm{lb} / \mathrm{s}$ $(106.6 \mathrm{~kg} / \mathrm{s})$. The total pressure recovery is calculated by massaveraged integration at the AIP. The TPR reaches its maximum value when the curved normal shock wave hits the cowl lip and no secondary normal shock occurs, as shown in Fig. $8 \mathrm{~b}$. The maximum total pressure recovery of $96 \%$ is consistent with other axisymmetric computation for the same configuration [13]. As the backpressure at the engine fan face is reduced another normal shock wave appears and moves downstream in the core path, and the mass-flow rate is increased accordingly until the flowpath is choked as in Fig. 8a.

For all the solution points on the cane curve, the bypass duct is choked: the flow in the bypass duct begins with subsonic speed and accelerates continuously to supersonic speed through a throat point, without being terminated by a shock wave in the bypass duct.

The contours of normalized total pressure in Fig. 9 show one of demerits of the relaxed compression inlet: the reduced cowl angle causes a rapid turning angle at the center body shoulder region, which causes a thicker boundary layer in the subsonic diffuser. Also comparing Figs. $9 \mathrm{~b}$ and $9 \mathrm{c}$, one can note that most portion of the flow with less pressure recovery because of the stronger terminal shock wave in Fig. 9c is being dumped out through the bypass duct so that degradation of the total pressure recovery at the AIP is minimized. This fact is shown more clearly in Fig. 10.

Figure 10 shows variation of total pressure recovery and massflow rate at the entrance of bypass duct as the core mass-flow rate at the AIP changes. From Fig. 10a, it can be noted that the total pressure recovery of the bypass flow also becomes maximum when the core total pressure recovery is maximum. The combined TPR of the core and bypass passages corresponds to TPR of a conventional singlepassage inlet. The core TPR is less sensitive to the change of core mass-flow rate than the bypass and combined TPR, which is another merit of the bypass inlet. Figure $10 \mathrm{~b}$ shows that the mass-flow rate through the bypass duct is proportional to the core mass-flow rate and, moreover, that the bypass duct and core flows are interconnected.

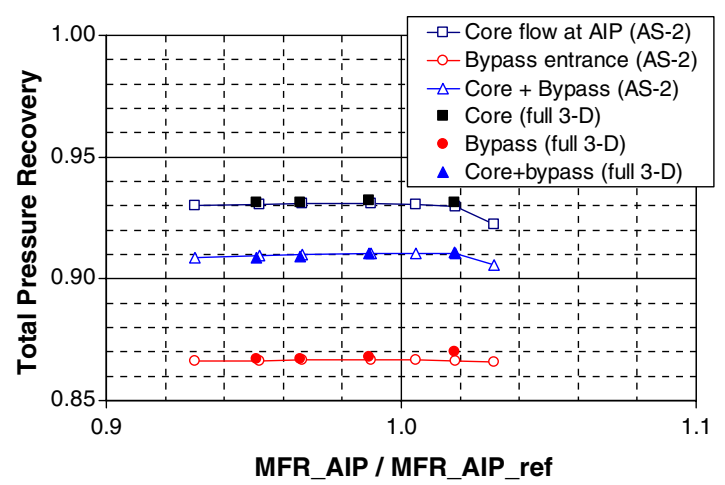

a) Total pressure recovery at AIP and bypass entrance

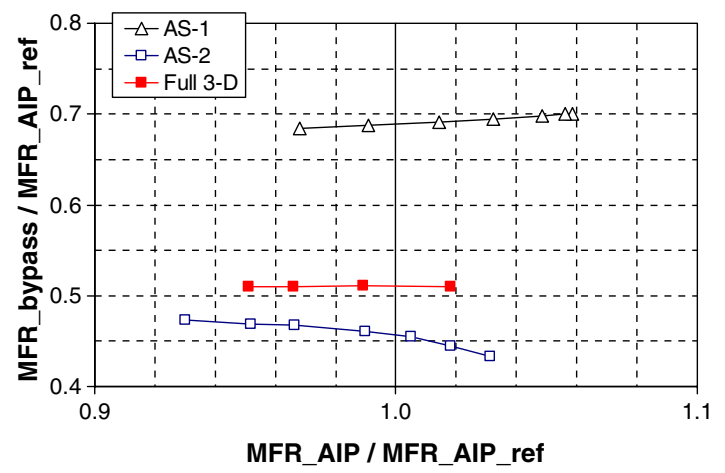

b) Relation between mass flow rates at AIP and bypass duct

Fig. 14 Comparison of mass-flow rate and total pressure recovery at entrance of the bypass duct for AS-2 and full three-dimensional configurations. 


\section{B. Full Three-Dimensional Configuration: Inlet Performance}

In this section, the full three-dimensional configuration with struts and fairing is considered. First, approximate cane curves for the full three-dimensional configuration is generated using axisymmetric simulations with the blockage matching approach described in Sec. III.D. The blockage is matched at the normalized backpressure

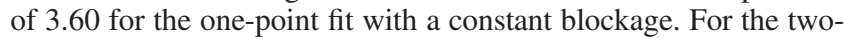
point fit, another point with the normalized backpressure of 3.70 is added to build a linear blockage model.

The approximate cane curves by the one- and two-point fits of the blockage are shown in Fig. 11. These two cane curves cross each other at the normalized pressure of 3.60 and deviate at smaller and larger backpressures. For the linear blockage model, the blockage in the bypass duct appears proportional to the core mass-flow rate. Comparison of solution points for AS-2 and full three-dimensional configurations, denoted by the symbols wrapped in green bags in Fig. 11, shows that the linear blockage fit is much more accurate than the constant fit at both $P_{b} / P_{\infty}=3.664$ and 3.40 and provides a very close performance approximation to the full three-dimensional configuration. This fact suggests that the present assumption on the bypass blockage is valid and that the flow analyses are expected to be reliable and accurate.

Some representative solutions for AS-2 with the two-point fit are visualized in terms of Mach contours in Fig. 12. All the AS-2 results in the remaining of this paper refer to those with the two-point fit. A curved normal shock wave is standing upstream of the cowl lip due to the blockage in the bypass duct. For the choked condition, another normal shock appears in the core path, as depicted in Fig. 12a. As the backpressure increases, the terminal normal shock in front of the cowl lip slowly moves farther upstream.

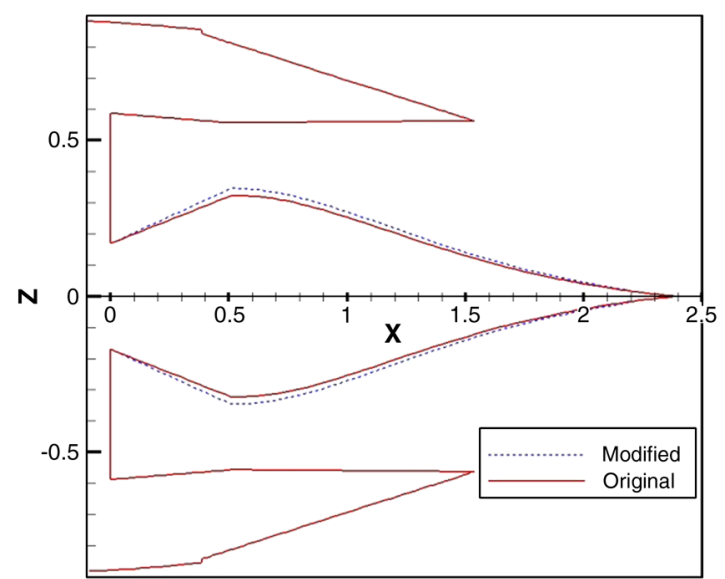

Fig. 15 Comparison of original and modified plug nozzle geometries for matching the engine exhaust mass-flow rate at the inlet-engine operating condition.
Table 1 Axial force of the full three-dimensional bypass engine nacelle at the inlet-engine operating condition

\begin{tabular}{lc}
\hline \hline Force terms & Value, № \\
\hline Pressure force on AIP & 33,294 \\
Pressure force on engine exhaust & $-73,938$ \\
Pressure force by other components & 32,740 \\
Momentum through AIP & 20,765 \\
Momentum through exhaust & $-17,278$ \\
Friction force & 3,308 \\
Total sum & $-1,109$ \\
\hline \hline
\end{tabular}

${ }^{\mathrm{a}} \mathrm{A}$ negative value means thrust force.

Mach contours for the full three-dimensional configuration at normalized backpressures of 3.50, 3.60, and 3.70 are shown in Fig. 13 , which are very similar to the contours for AS-2 represented in Fig. 12 .

Figure 14 shows the variation of total pressure recovery and massflow rate at the entrance of bypass duct as the core mass-flow rate at the AIP changes. Overall, the total pressure recoveries of AS-2 and full three-dimensional configurations compare very well, as can be seen in Fig. 14a. It is also noted that the total pressure recovery of the bypass flow is almost constant for the variation of the core mass-flow rate. Figure $14 \mathrm{~b}$ shows that the bypass mass-flow rate of AS-2 is lower than that of full three-dimensional configuration and inversely proportional to the core mass-flow rate. Meanwhile, $\mathrm{MFR}_{\text {bypass }}$ is proportional to $\mathrm{MFR}_{\mathrm{AIP}}$ for AS-1 and almost constant for the full three-dimensional configuration. The discrepancy in $\mathrm{MFR}_{\text {bypass }}$ for AS-2 is in contrast to the good agreements of $\mathrm{MFR}_{\mathrm{AIP}}, \mathrm{TPR}_{\mathrm{AIP}}$, and $\mathrm{TPR}_{\text {bypass }}$ between AS-2 and full three-dimensional configurations. The direct cause of the inversely proportional variation of the $\mathrm{MFR}_{\text {AIP }}$ with respect to $\mathrm{MFR}_{\mathrm{AIP}}$ is the linear blockage model, which is set as proportional to $\mathrm{MFR}_{\mathrm{AIP}}$ in order to match inlet core performances.

\section{Full Three-Dimensional Configuration: Coupled Performance of Inlet Engine}

Now we consider a coupled analysis of the inlet and engine for performance evaluation of the whole bypass engine nacelle. The coupled operation condition for inlet engine can be determined at a point where the cane curve intersects the TPR vs MFR line in Fig. 6 a of the engine model (see Fig. 11). The same point can be found by carrying out a flow simulation of the full three-dimensional geometry with the boundary conditions from the engine model.

The red triangle in Fig. 11 is the inlet operating point for the inletengine matching at the supersonic cruise flight condition. It almost coincides with the intersection point between the approximate cane curve of AS-2 with the two-point fit and the engine mass-flow line.

At the inlet-engine coupled condition, the total pressure recovery is $93.16 \%$, and mass-flow rate is $102.95 \mathrm{~kg} / \mathrm{s}$. The engine exhaust mass-flow rate can be matched to a target value by adjusting the

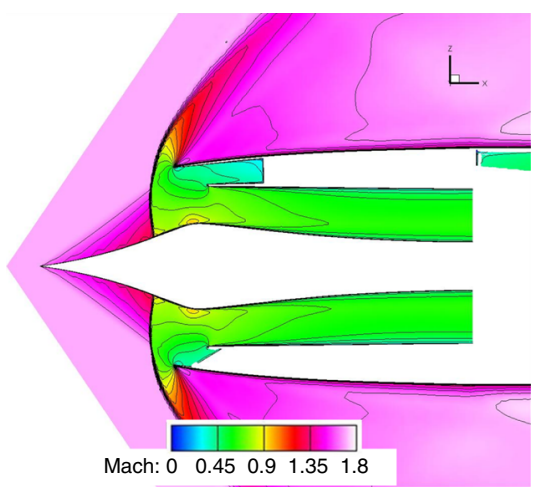

a) Mach contours of inlet

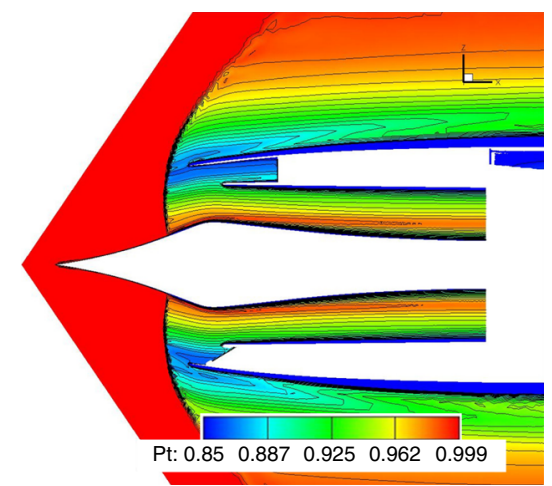

b) Total pressure contours of inlet

Fig. 16 Results of the full three-dimensional configuration at the inlet-engine operating condition: inlet region. 


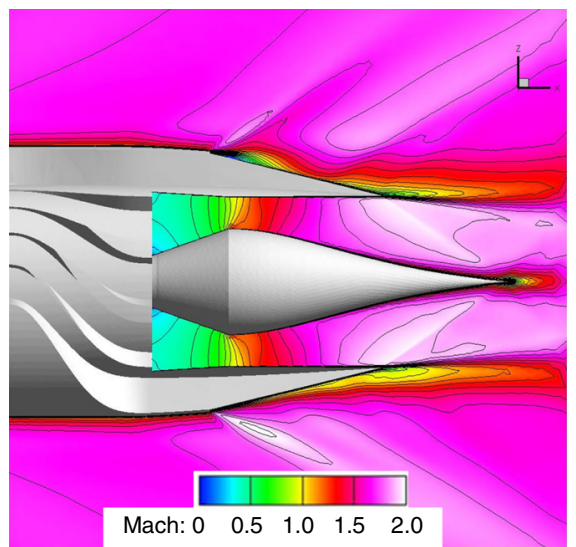

Fig. 17 Results of the full three-dimensional configuration at the inletengine operating condition: Mach contours near engine exhaust.

throat area of the plug nozzle, as shown in Fig. 15. The total axial force of the full three-dimensional bypass nacelle is calculated by the surface integration over viscous walls and engine face/exhaust planes. The resulting axial force presented in Table $\underline{1}$ shows that the bypass nacelle generates thrust.

Some flow features are visualized for the simulation results at the inlet-engine coupled condition. In Fig. 16, Mach and total pressure contours are shown in the near-inlet region. The additional blockage in the complex passages of the bypass duct causes the terminal normal shock to move farther upstream of the cowl lip compared to AS-1 shown previously in Sec. IV.A. The converging-diverging plug nozzle accelerates the subsonic flow exhausted by the engine to supersonic speed, as evident in Fig. 17.

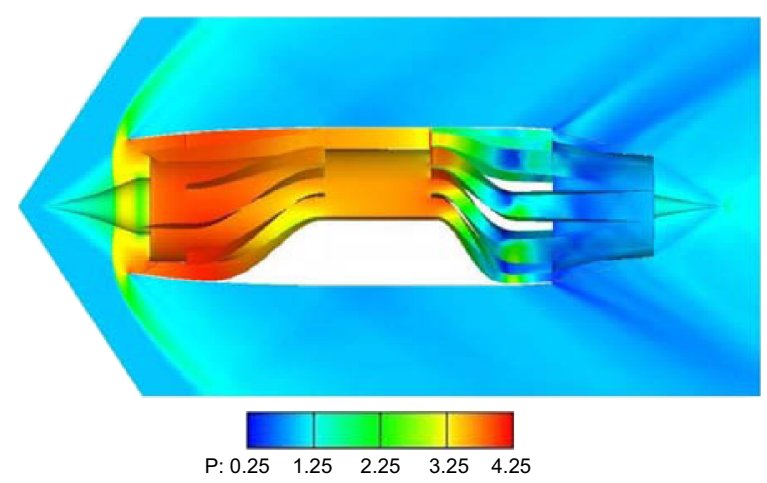

a) Full three-dimensional configuration

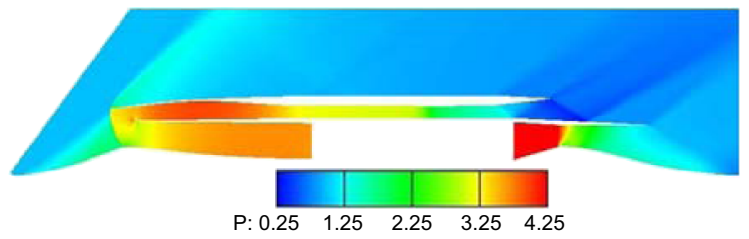

b) AS-1 configuration

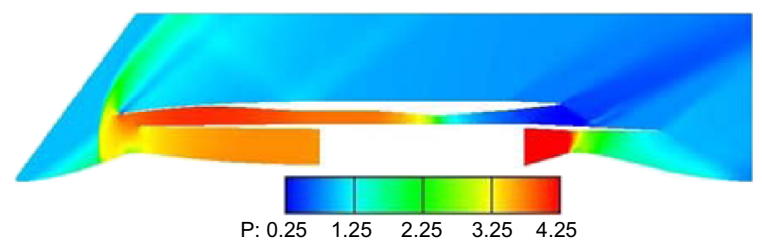

c) AS-2 configuration

Fig. 18 Results of the full three-dimensional configuration at the inletengine operating condition: overall pressure contours. Pressure contours of AS-1 and AS-2 with the same normalized backpressure (3.664) are shown for comparison.

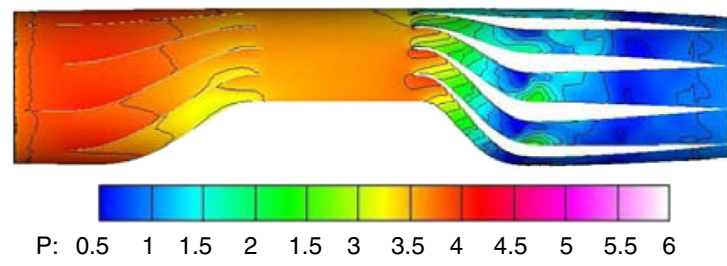

Fig. 19 Results of the three-dimensional configuration with faring and struts at the inlet-engine operating condition: pressure contours on the bypass duct inner surface (flow from left to right).

The overall pressure contours for the full three-dimensional configuration at the inlet-engine coupled condition are displayed in Fig. 18. The normalized backpressure for the inlet-engine operating condition is 3.664. In Fig. 18, results of AS-1 and AS-2 configurations with the same backpressure are also compared. The AS-1

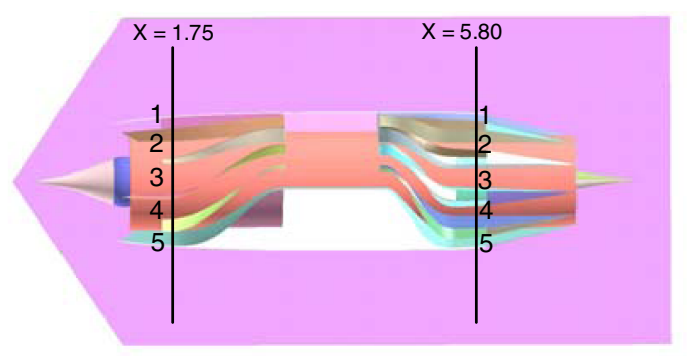

a) Location and indexing of flow passages in the bypass duct

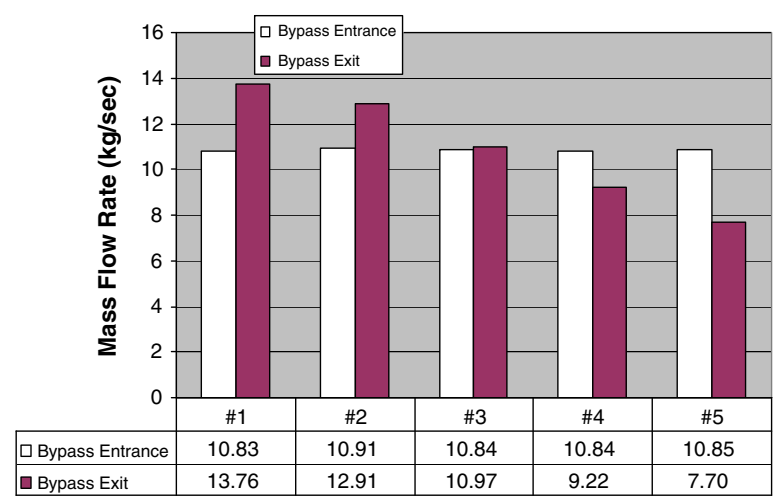

b) Comparison of mass flow rates at entrance and exit of the bypass duct

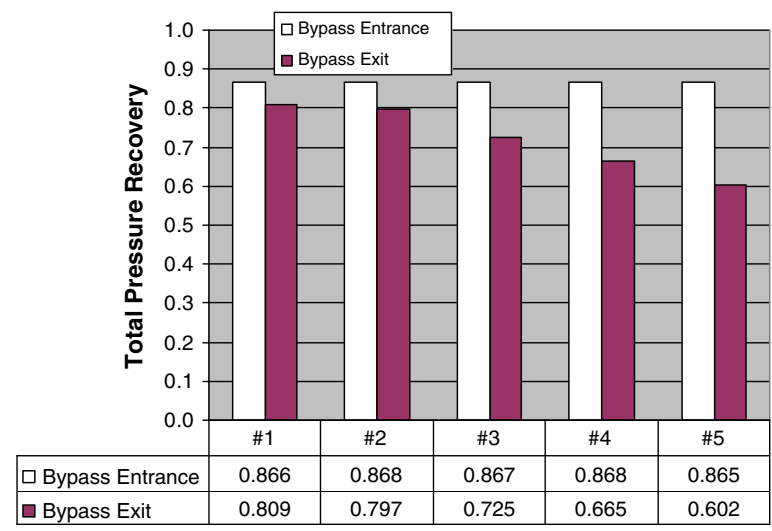

c) Comparison of total pressure recoveries at entrance and exit of the bypass duct

Fig. 20 Flow quality through the bypass duct at the inlet-engine operating condition. 


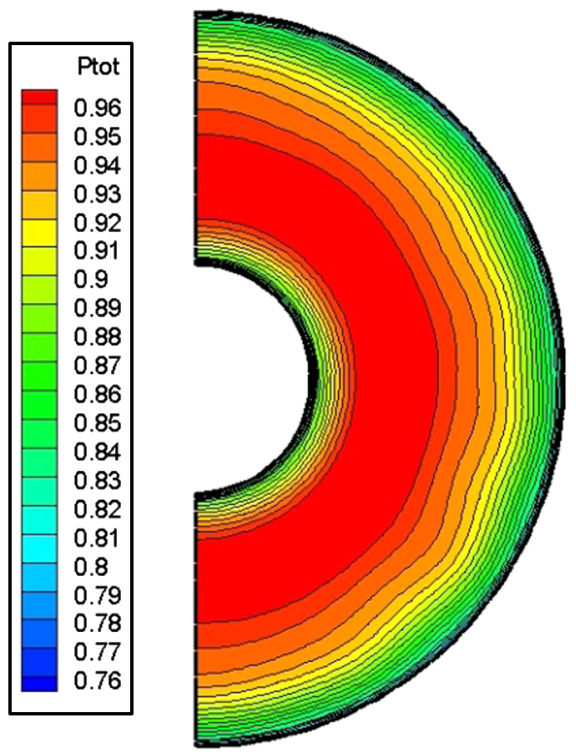

Fig. 21 Total pressure contours at the AIP.

configuration shows a totally different terminal shock location from the full three-dimensional configuration. Meanwhile, for the full three-dimensional and AS-2 configurations, the overall trends of the contours show a good agreement, from the inlet shock pattern through the bypass duct and to the exit of the nozzle.

A region of great interest is inside the bypass duct. As shown in Fig. 19, the front and mid parts of the bypass duct show smooth flow patterns. However, in the rear part of the duct, where the flow is accelerated and guided by the curved struts, oblique shocks are occurring in a couple of flow passages having severe curvature. The abrupt change in flowpath near the fairing produces shock waves, and subsonic pockets embedded in the supersonic flow seems to be the main reason of mass-flow spillage at the inlet.

The flow quality in the bypass duct is further investigated by checking the mass-flow rate and total pressure recovery at the entrance of the front five passages and exit of the rear five passages. The flow entering the bypass duct is guided by the five front passages and then merges together before entering the rear passages in the diverging part. Figure 20a shows the definition for indexing the front and rear five passages. Figures $20 \mathrm{~b}$ and $20 \mathrm{c}$ compare the mass-flow rate and total pressure recovery at each passage. Overall, the entering flow is well distributed into five passages and total pressure recovery is also almost uniform. However, at the exit of the duct, the flow rate and total pressure recovery decrease as the flow passage closes to the fairing, which means more curved flowpaths. An optimal shape design of the flowpath in the diverging region to reduce occurrence of shock waves and boundary-layer thickness would lead to a much reduced flow blockage and thus substantial improvement to the bypass inlet performance.

Flow distortion at the AIP is visualized with contours of normalized total pressure in Fig. 21 at the engine-inlet matching condition. Although the inlet geometry is axisymmetric, the total pressure contours are slightly deviated from axisymmetry because of the front struts in the bypass duct. Since the normal shock is sitting upstream of cowl lip, as shown in Fig. 16, existence of struts are affecting the core path down to the AIP.

Table 2 Comparison of inlet performance for original and fine grids at inlet-engine matching condition

\begin{tabular}{lccc}
\hline \hline & Original & Fine grid & $\Delta, \%$ \\
\hline Number of nodes & $7,214,163$ & $17,894,278$ & - \\
MFR, $\mathrm{kg} / \mathrm{s}$ & 102.95 & 103.35 & 0.37 \\
TPR, $\%$ & 93.16 & 93.49 & 0.35 \\
\hline \hline
\end{tabular}

\section{Full Three-Dimensional Configuration: Grid Refinement Study}

Here, we present a grid refinement study to show our grid density is sufficient to accurately capture flow quality of the threedimensional supersonic bypass inlet. A very fine grid is generated with $17,894,278$ nodes, which is 2.5 times as many grid points as the original grid. As shown in Table 2, the fine grid results in slightly higher TPR and MFR at the engine AIP because of a slightly less blockages effect. This is a result of lower numerical dissipation from the finer computational grid. However, the differences of inlet performances at the inlet-engine matching condition are very small. The contour plots of aerodynamic variables also show very slight differences from each other and hence are not displayed here.

\section{Conclusions}

Flow simulations were conducted for the supersonic bypass inlet configuration proposed for reduced sonic boom and wave drag from the inlet cowl. The flowfield around the complex geometry including gearbox fairing and struts was solved using the Reynolds-averaged Navier-Stokes equations on unstructured hybrid grids. Axisymmetric and full three-dimensional configurations were considered to investigate flow physics and evaluate performance of the configuration. Inlet-engine coupling was made by interactively imposing engine face and exhaust boundary conditions with the NPSS engine model, such that the required mass-flow rate is delivered to the engine. An efficient approach was suggested for building approximate cane curves for bypass inlets by using axisymmetric simulations with adjusted bypass blockage. Simulation results show that the flow quality through the bypass duct is deteriorated near the gearbox fairing because of the severe variation of flow passages. It was found that the increased blockage in the bypass duct substantially degrades the supersonic inlet performance at the core path.

As a future work, an optimal shape design of flow passages in the bypass duct will be conducted to reduce bypass blockage and improve the inlet performance.

\section{Acknowledgements}

The authors are grateful for the support by the NASA's Supersonics Project of Fundamental Aeronautics Program. Gulfstream Aerospace Corporation supplied the bypass duct geometry.

\section{References}

[1] Conners, T. R., and Howe, D. C., "Supersonic Inlet Shaping for Dramatic Reduction in Drag and Sonic Boom Strength," 44th AIAA Aerospace Science Meeting and Exhibit, AIAA Paper 2006-0030, Reno, NV, Jan. 2006.

[2] Hirt, S. M., Tacina, K. M., Conners, T. R., Merret, J. M., and Howe, D. C., "CFD Results for an Axisymmetric Isentropic Relaxed Compression Inlet," 46th AIAA Aerospace Science Meeting and Exhibit, AIAA Paper 2008-0092, Reno, NV, Jan. 2008.

[3] Nakahashi Ito, Y., and Togashi, F., "Some Challenges of Realistic Flow Simulations by Unstructured Grid CFD," International Journal for Numerical Methods in Fluids, Vol. 43, Nos. 6-7, 2003, pp. 769-783.

[4] Venkatakrishnan, V., "On the Accuracy of Limiters and Convergence to Steady State Solutions," AIAA Paper 93-0880, 1993.

[5] Obayashi, S., and Guruswamy, G. P., "Convergence Acceleration of a Navier-Stokes Solver for Efficient Static Aeroelastic Computations," AIAA Journal, Vol. 33, No. 6, 1995, pp. 1134-1141. doi: $10.2514 / 3.12533$

[6] Spalart, P. R., and Allmaras, S. R., "A One-Equation Turbulence Model for Aerodynamic Flows," Recherche Aerospatiale, Vol. 1, 1994, pp. 5-21.

[7] Sharov, D., and Nakahashi, K., "Reordering of Hybrid Unstructured Grids for Lower-Upper Symmetric Gauss-Seidel Computations," AIAA Journal, Vol. 36, No. 3, 1998, pp. 484-486. doi: $10.2514 / 2.392$

[8] Ito, Y., and Nakahashi, K., "Direct Surface Triangulation Using Stereolithography Data," AIAA Journal, Vol. 40, No. 3, March 2002, pp. 490-496. doi:10.2514/2.1672 
[9] Ito, Y., Shih, A. M., Soni, B. K., and Nakahashi, K., "Multiple Marching Direction Approach to Generate High Quality Hybrid Meshes," AIAA Journal, Vol. 45, No. 1, Jan. 2007, pp. 162-167. doi: $10.2514 / 1.23260$

[10] Russell, W. C., Townsend, S., Lavelle, T., Naiman, C., and Turner, M., "A Case Study of High Fidelity Engine System Simulation," 42nd AIAA/ASME/SAE/ASEE Joint Propulsion Conference \& Exhibit, AIAA Paper 2006-4971, Sacramento, CA, July 2006.

[11] O’Brien, D. M., Jr., Calvert, M. E., and Butler, S. L., "An Examination of Engine Effects on Helicopter Aeromechanics," AHS Specialist's
Conference on Aeromechanics, AHS International, Alexandria, VA, Jan. 2008.

[12] Anderson, J. D., Modern Compressible Flow: with Historic Perspective, 3rd ed., McGraw-Hill, New York, 2003, p. 216.

[13] Chima, R., Conners, T., and Wayman, T., "Coupled Analysis of an Inlet and Fan for a Quiet Supersonic Aircraft," AIAA Aerospace Science Meeting and Exhibit, AIAA Paper 2010-0479, Orlando, FL, Jan. 2010

R. Bowersox Associate Editor 